\title{
Comparison of Aerocapture Performance Using Bank Control and Direct Force Control with Two Human-Scale Vehicles at Mars
}

\author{
Daniel A. Matz*, Christopher J. Cerimele ${ }^{\dagger}$, and Ronald R. Sostaric ${ }^{\ddagger}$ \\ NASA Johnson Space Center, Houston, TX 77058
}

\begin{abstract}
Recent studies of human-scale missions to Mars have included a wide trade space of vehicle configurations and control schemes. Some configurations fly at a low angle of attack with a low $\mathrm{L} / \mathrm{D}$, while others fly at a high angle of attack with a mid L/D. Some use bank angle control, while others use direct force control, where the angle of attack and sideslip angle are independently modulated. This paper compares the performance of three vehicle configurations: a low-L/D vehicle using direct force control, a low-L/D vehicle using bank control, and a mid-L/D vehicle using bank control. The reference mission is aerocapture at Mars into a highly elliptical, 1-sol orbit. The trajectories are integrated in three degrees of freedom. All three cases utilize numeric predictor-corrector guidances and emulate control system responses with rate and acceleration limits. The configurations are compared using a Monte Carlo analysis. The robustness of each configuration to increased dispersions is also compared.
\end{abstract}

\section{Introduction}

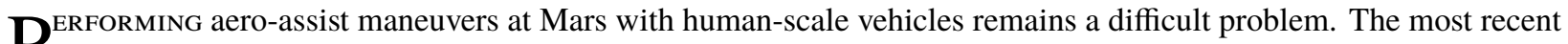

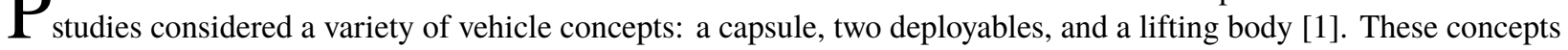
feature different ballistic coefficients, lift-to-drag (L/D) ratios, and control schemes.

Some of the concepts propose the use of bank angle control, which is a proven technique that has been used by many vehicles both at Earth and at Mars. By modulating the bank angle, the direction of the lift force can be controlled. However, the longitudinal and lateral guidance channels are inherently coupled, necessitating the use of bank reversals.

Other concepts instead propose the use of a new control scheme called direct force control (DFC) [2]. This approach modulates the angle of attack and sideslip angle independently, which decouples the longitudinal and lateral channels. However, the constraints on the angle of attack and sideslip angle variation may result in less control authority in the longitudinal and lateral channels than the bank angle approach can provide.

In this paper, we will compare the performance and robustness of two vehicle configurations using bank angle control and direct force control. The first vehicle configuration has an $\mathrm{L} / \mathrm{D}$ of 0.15 , a ballistic coefficient of $165 \mathrm{~kg} / \mathrm{m}^{2}$, and a low trim angle of attack. The second has an L/D of 0.54 , a ballistic coefficient of $395 \mathrm{~kg} / \mathrm{m}^{2}$, and a trim angle of attack of $55^{\circ}$. Direct force control isn't practical for vehicles flying at high angles of attack, because the angle of attack would need to be modulated over a range of $100^{\circ}$ or more. Thus, we are left with three cases: the low-L/D vehicle using direct force control, the low-L/D vehicle using bank control, and the mid-L/D vehicle using bank control.

\section{Simulation Configuration}

The Flight Analysis and Simulation Tool (FAST) was used to generate dispersed, three-degree-of-freedom trajectories. Atmospheric properties were computed using Mars Global Reference Atmospheric Model (GRAM) 2010. The reference mission is aerocapture at Mars into a highly elliptical, 1-sol orbit, specified in Table 1 The initial conditions for the study are given in Table 2 .

Both control approaches use an appropriate numeric predictor-corrector guidance and have emulated, finite-rate attitude responses. The following sections briefly summarize the two guidance schemes used.

\footnotetext{
*Aerospace Engineer, Flight Mechanics and Trajectory Design Branch, AIAA Senior Member

${ }^{\dagger}$ Chief (retired), Flight Mechanics and Trajectory Design Branch, AIAA Senior Member

$\doteqdot$ Aerospace Engineer, Flight Mechanics and Trajectory Design Branch, AIAA Senior Member
} 
Table 1 Target orbit.

\begin{tabular}{lc}
\hline Radius of Apoapsis & $33793 \mathrm{~km}$ \\
Radius of Periapsis & $250 \mathrm{~km}$ \\
Inclination & $90^{\circ}$ \\
Longitude of Ascending Node & $180^{\circ}$ \\
\hline
\end{tabular}

Table 2 Initial conditions.

\begin{tabular}{lc}
\hline Latitude & $82^{\circ}$ \\
Longitude & $0^{\circ}$ \\
Planetodetic Altitude & $125 \mathrm{~km}$ \\
Inertial Velocity & $6.2 \mathrm{~km} / \mathrm{s}$ \\
Planetodetic Inertial Azimuth & $180^{\circ}$ \\
\hline
\end{tabular}

\section{A. Bank Control}

Both bank control configurations use the Fully Numerical Predictor-corrector Aerocapture Guidance (FNPAG) algorithm [3]. FNPAG is based on the optimal solution to the aerocapture problem using bank angle control, which dictates a bang-bang solution, where the vehicle first flies full lift up and then at some point switches to full lift down. The guidance consists of two phases. In the first phase, the predictor uses the profile shown in Figure 1, where $\phi_{0}$ and $\phi_{d}$ are specified by the user. The corrector iterates to solve for the value of the switching time, $t_{s}$, such that the predicted radius of apoapsis is equal to the provided target. Once $t_{s}$ is reached, the second phase begins, during which the predictor uses a constant bank angle command and the corrector solves for the value of that command which achieves the radius of apoapsis target.

Because the longitudinal and lateral channels are coupled, we must use bank reversals for lateral control. We use an additional predicted trajectory to perform a user-specified number of bank reversals to geometrically reduce the wedge angle, which is the angle between the instantaneous angular momentum vector and the vector normal to the target orbital plane [4]. Both bank control configurations in this study command five bank reversals.

While the trajectories are only integrated in three degrees of freedom, we do emulate the attitude kinematics. We assume that bank angle is controlled by an RCS system. We use a finite-state machine to emulate a bang-bang control effector subject to a bank angle rate limit of $15 \% \mathrm{~s}$ and a bank angle acceleration limit of $5 \% \mathrm{~s}^{2}$. The angle of attack is held constant at an appropriate trim value. For the mid-L/D vehicle, the trim angle of attack is $55^{\circ}$, and for the low-L/D vehicle, the trim angle of attack is $-9.86^{\circ}$.

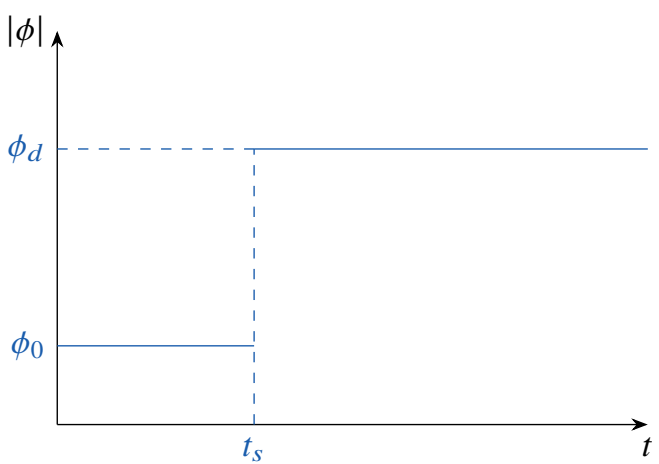

Fig. 1 Bank profile used in the first phase. 


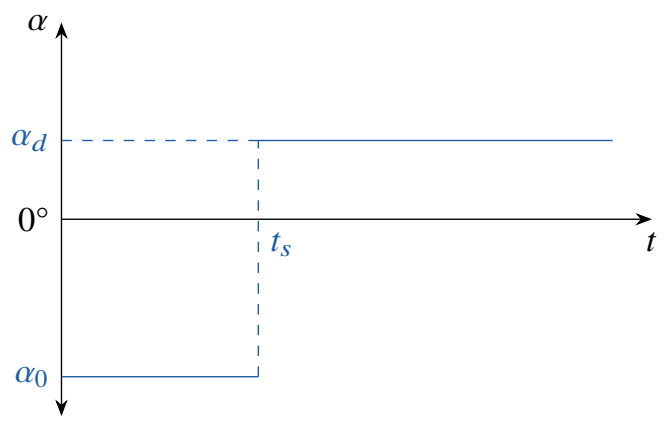

Fig. 2 Angle of attack profile used in the first phase.

Table 3 Limits placed on the rotational kinematics for direct force control.

\begin{tabular}{lccc}
\hline & $\begin{array}{c}\text { Angle Limit } \\
{\left[{ }^{\circ}\right]}\end{array}$ & $\begin{array}{c}\text { Rate Limit } \\
{[\% / \mathrm{s}]}\end{array}$ & $\begin{array}{c}\text { Accel Limit } \\
{\left[\% / \mathrm{s}^{2}\right]}\end{array}$ \\
\hline Angle of Attack $(\alpha)$ & \pm 9.51 & 2.5 & 1.0 \\
Sideslip Angle $(\beta)$ & \pm 5 & 2.5 & 1.0 \\
\hline
\end{tabular}

\section{B. Direct Force Control}

When using direct force control, the longitudinal and lateral channels are largely decoupled. If we fix the bank angle at $0^{\circ}$, then the angle of attack is used for the longitudinal channel and the sideslip angle is used for the lateral channel.

A new version of FNPAG for use with direct force control has been developed [5]. Similar to FNPAG for bank angle control, the guidance uses a numeric predictor-corrector with a two-phase structure in the longitudinal channel. In the first phase, the predictor uses the profile shown in Figure 2, where $\alpha_{0}$ and $\alpha_{d}$ are specified by the user, to compute the radius of apoapsis when leaving the atmosphere. The corrector solves for the required value of $t_{s}$ such that the predicted trajectory achieves the desired radius of apoapsis. When that time is reached, the guidance moves to the second phase, where it finds a constant angle of attack command in order to attain the specified radius of apoapsis. For the lateral channel, a simple proportional-derivative (PD) controller is used to command the sideslip angle. The controller works to null the position and velocity components perpendicular to the targeted orbital plane.

The angle of attack and sideslip angle are assumed to be controlled using continuous commands. The effector is not specified, but could be something like a flap or movable center of mass. The responses of angle of attack and sideslip angle are emulated using PD controllers subject to the rate and acceleration limits shown in Table 3.

\section{Monte Carlo Results}

For each configuration, a 1000-case Monte Carlo was generated using the dispersions summarized in Table 4. For the mid-L/D vehicle, the nominal planetodetic inertial flight path angle was $-10.1^{\circ}$. For the low-L/D vehicle, both cases used a nominal flight path angle of $-9.95^{\circ}$.

The configurations were compared in terms of two primary metrics: the total $\Delta V$ required to clean up the orbit after the aerocapture maneuver and the peak sensed acceleration experienced during the maneuver. The $\Delta V$ is computed analytically from the conditions at atmospheric exit and includes three burns. First, the vehicle performs a burn at apoapsis to raise its periapsis out of the atmosphere. Second, it performs a burn at periapsis to correct any remaining error in the apoapsis. Lastly, it performs a burn to correct the orbital plane. No burn is performed to correct the line of apsides.

The Monte Carlo results are co-plotted in Figure 3 All three cases performed well, with no cases failing to capture. The $\Delta V$ results are fairly similar across the three cases and are summarized in Table 5. All three cases resulted in relatively low peak sensed accelerations. The mid-L/D vehicle experienced the lowest peak sensed accelerations. 
Table 4 Monte Carlo dispersions.

\begin{tabular}{lc}
\hline Variable & Dispersion \\
\hline GRAM Random Seed & uniform, 1-29999 \\
GRAM Dust $\tau$ & uniform, $0.1-0.9$ \\
Mass & normal, $\sigma=0.3333 \%$ \\
Aero Coefficients & uniform, $\pm 10 \%$ \\
Initial Longitude & normal, $\sigma=0.08333^{\circ}$ \\
Initial Planetodetic Latitude & normal, $\sigma=0.08333^{\circ}$ \\
Initial Planetocentric Inertial Flight Path Angle & normal, $\sigma=0.03333^{\circ}$ \\
Initial Planetocentric Inertial Azimuth & normal, $\sigma=0.08333^{\circ}$ \\
Initial Inertial Velocity & normal, $\sigma=3.333 \mathrm{~m} / \mathrm{s}$ \\
\hline
\end{tabular}
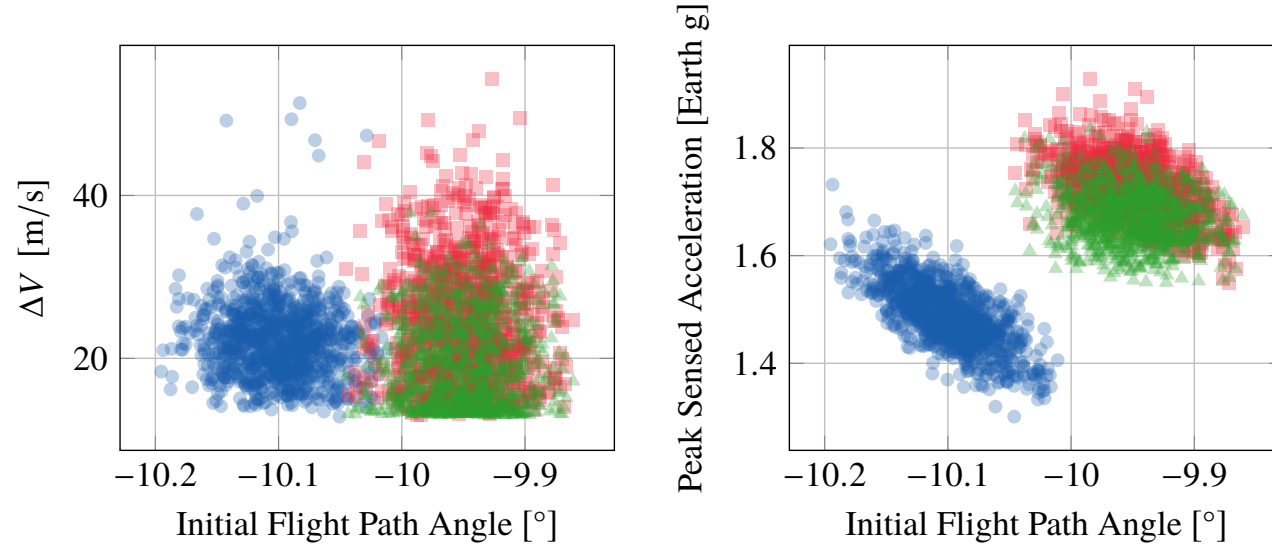

- Mid L/D, Bank Low L/D, Bank Low L/D, DFC

Fig. 3 Comparison of Monte Carlo results.

Table 5 Comparison of required post-aerocapture $\Delta V$.

\begin{tabular}{lcc}
\hline & $\begin{array}{c}\text { Mean } \Delta V \\
{[\mathrm{~m} / \mathrm{s}]}\end{array}$ & $\begin{array}{c}\text { Mean }+3 \sigma \Delta V \\
{[\mathrm{~m} / \mathrm{s}]}\end{array}$ \\
\hline Mid L/D, Bank & 22 & 36 \\
Low L/D, Bank & 25 & 47 \\
Low L/D, DFC & 19 & 36 \\
\hline
\end{tabular}



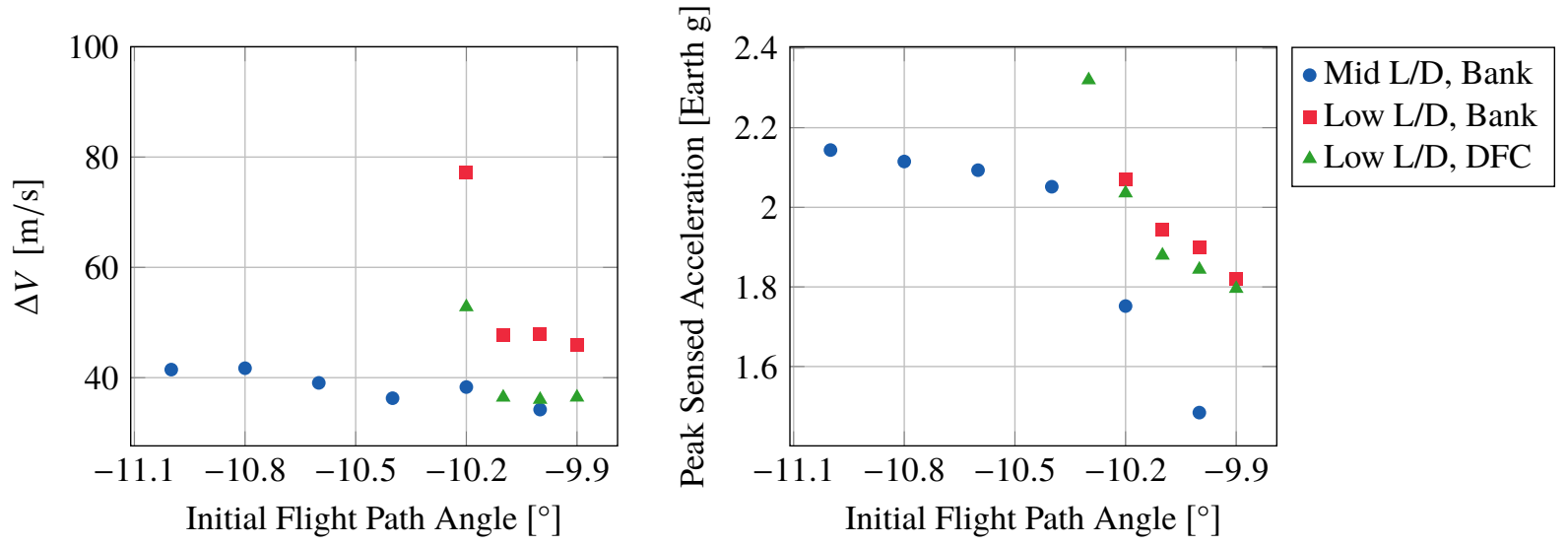

Fig. 4 Flight path angle robustness scan.

Table 6 Summary of the robustness scans.

\begin{tabular}{lccc}
\hline Dispersion & Mid L/D, Bank & Low L/D, Bank & Low L/D, DFC \\
\hline EI Flight Path Angle & $-11.0^{\circ}$ to $-10.0^{\circ}$ & $-10.2^{\circ}$ to $-9.8^{\circ}$ & $-10.2^{\circ}$ to $-9.8^{\circ}$ \\
EI Azimuth Angle & $-2.7^{\circ}$ to $+2.5^{\circ}$ & $-1.0^{\circ}$ to $+0.9^{\circ}$ & $-0.5^{\circ}$ to $+1.4^{\circ}$ \\
$C_{D}$ Dispersion Multiplier & $5.2 \times$ & $3.0 \times$ & $3.3 \times$ \\
$C_{L}$ Dispersion Multiplier & $3.7 \times$ & $4.7 \times$ & $4.5 \times$ \\
GRAM Dispersion Multiplier & $>2.0 \times$ & $1.7 \times$ & $>2.0 \times$ \\
Mass Dispersion Multiplier & $45 \times$ & $32 \times$ & $35 \times$ \\
\hline
\end{tabular}

\section{Robustness Study}

Additional Monte Carlo simulations were performed to assess the robustness of the three configurations to increased dispersions. In general, the methodology was to apply a multiplier to the dispersion of interest while dispersing the other variables as usual. For each value of the multiplier, a Monte Carlo was performed. The goal was to find the largest value of the multiplier that would still result in acceptable performance. For our purposes, an acceptable Monte Carlo was one with no cases that failed to capture, a mean $+3 \sigma \Delta V$ less than $50 \mathrm{~m} / \mathrm{s}$, and a mean $+3 \sigma$ peak sensed acceleration less than 4 Earth gs.

The multiplier approach was used for the $C_{L}, C_{D}$, mass, and GRAM dispersions. For the GRAM dispersions, the rpscale and rwscale parameters were both set to the value of the GRAM multiplier. These parameters are gains on the density and wind perturbations, respectively, and a value of 1.0 corresponds to a $3 \sigma$ perturbation level. The dust $\tau$ dispersion was left unchanged.

A slightly different approach was used for the flight path angle and azimuth angle dispersions. For those two cases, the scan was performed on the angle itself with no dispersion, but with all the other dispersions applied as usual, resulting in what is essentially a dispersed corridor width assessment.

The same guidance configuration was used throughout a scan. No guidance parameters were changed to attempt to compensate for the increased dispersions.

Figures 4 and 5 show examples of the data generated. In the figures, each dot represents the $3 \sigma$ value from a Monte Carlo. The results of all of the scans are summarized in Table 6 The higher L/D of the mid-L/D vehicle results in significantly wider flight path angle and azimuth angle corridors. The mid-L/D vehicle is somewhat more robust to drag coefficient and mass dispersions, while the low-L/D vehicle with either control approach is somewhat more robust to lift coefficient dispersions. A limitation in Mars GRAM 2010 prevented the GRAM multiplier from being set higher than 2, but it appears that the mid-L/D vehicle using bank control and the low-L/D vehicle using direct force control are both somewhat more robust to atmospheric dispersions than the low-L/D vehicle using bank control. 

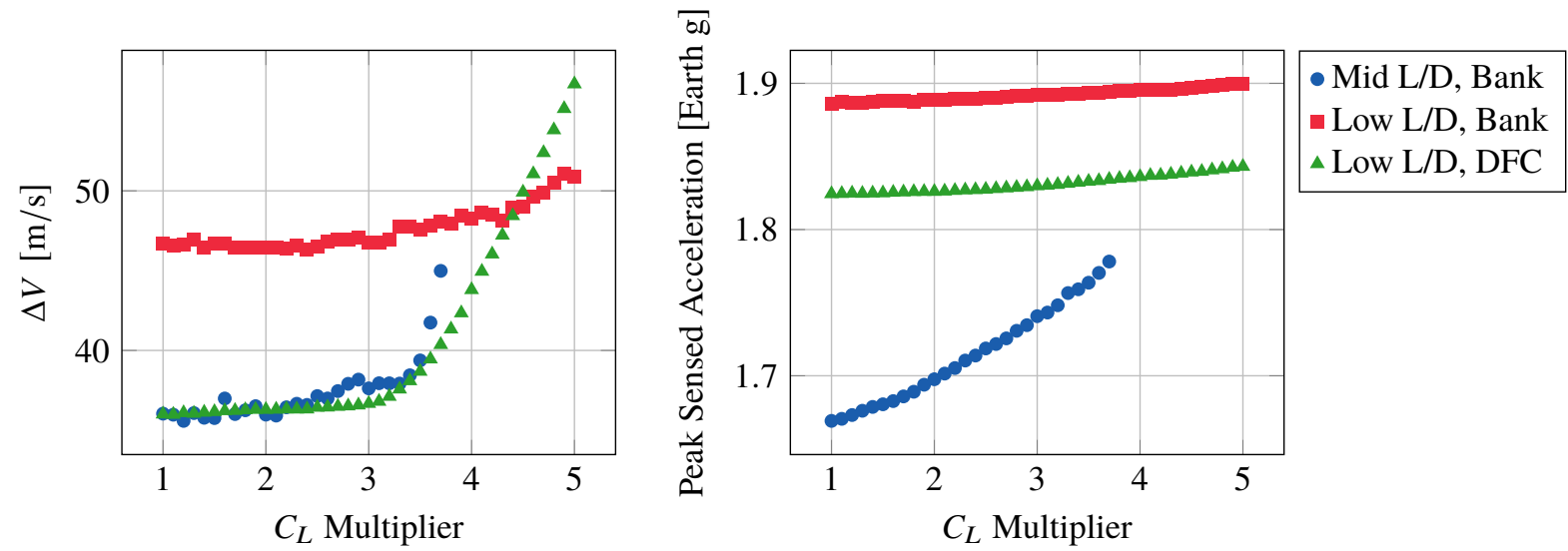

Fig. 5 Lift coefficient robustness scan.

\section{Conclusion}

Three human-scale Mars aerocapture vehicle and control configurations were compared using a Monte Carlo analysis. All three were able to perform the aerocapture maneuver without any cases failing to capture, with comparable required post-aerocapture $\Delta V$, and with peak sensed accelerations well below the limit of 4 Earth gs. The three cases were furthermore found to be robust to increases in dispersions. The most pronounced difference in robustness was the larger flight path angle and wedge angle corridors for the mid-L/D vehicle with bank control.

\section{References}

[1] Dwyer-Cianciolo, A. M., and Polsgrove, T., "Human Mars Entry, Descent, and Landing Architecture Study: Phase 2 Summary," 2018 AIAA SPACE and Astronautics Forum and Exposition, 2018. doi:10.2514/6.2018-5190, URL https: //doi.org/10.2514/6.2018-5190

[2] Dwyer-Cianciolo, A. M., and Powell, R. W., "Entry Descent and Landing Guidance and Control Approaches to Satisfy Mars Human Mission Landing Criteria,” AAS/AIAA Space Flight Mechanics Conference, 2017.

[3] Lu, P., Cerimele, C. J., Tigges, M. A., and Matz, D. A., "Optimal Aerocapture Guidance," AIAA Guidance, Navigation, and Control Conference, 2015. doi:10.2514/6.2015-1771, URL https://doi .org/10.2514/6.2015-1771.

[4] Smith, K. M., "Predictive Lateral Logic for Numerical Entry Guidance Algorithms," AAS/AIAA Space Flight Mechanics Meeting, 2016. URL https://ntrs.nasa.gov/search.jsp?R=20160001182

[5] Matz, D. A., and Cerimele, C. J., "Development of a Numeric Predictor-Corrector Aerocapture Guidance for Direct Force Control," AIAA Guidance, Navigation, and Control Conference, 2020. 